\title{
A Role of Fos Expression in the CA3 Region of the Hippocampus in Spatial Memory Formation in Rats
}

\author{
Jue He, M.D., Kiyofumi Yamada, Ph.D., and Toshitaka Nabeshima, Ph.D.
}

Activity-dependent plastic changes in the strength of synaptic connections are considered to underlie learning and memory. Activation of neurons by a variety of stimuli influences the level of expression of the immediate-early gene c-fos, and the heterodimer of the gene product, Fos, regulates transcription of other genes. Therefore, Fos is regarded as a mediator by which brief stimuli trigger longterm changes in the synaptic connections. Here we show that Fos expression in the CA3 region of the hippocampus may be obligatory for spatial memory formation in a radial arm maze test. Fos-positive cells increased in the cerebral cortices and the CA3 region of the dorsal hippocampus during the course of radial arm maze training in rats. Inhibition of Fos expression in this region of the hippocampus, but not the cingulate and motor cortex, by means of antisense oligonucleotide treatment resulted in an impairment of spatial memory formation. Our results support the hypothesis that the inducible transcription factor c-fos is essential for encoding spatial memory.

[Neuropsychopharmacology 26:259-268, 2002] (C) 2002 American College of Neuropsychopharmacology. Published by Elsevier Science Inc.
KEY WORDS: Learning and memory; Immediate-early genes; Synaptic plasticity; Hippocampus; Antisense oligonucleotide; c-Fos

Memory formation is considered to involve both shortterm and long-term changes in the strength of synaptic connections. Short-term changes may involve covalent modification of pre-existing proteins, whereas longterm changes in synaptic connections may be associated with synaptogenesis and neuropil growth (Burns and Augustine 1995; Edwards 1995; Kleim et al. 1996),

From the Department of Neuropsychopharmacology and Hospital Pharmacy, Nagoya University Graduate School of Medicine, Showa-Ku, Nagoya 466-8560, Japan

Address correspondence to: T. Nabeshima, Ph.D., Department of Neuropsychopharmacology and Hospital Pharmacy, Nagoya University Graduate School of Medicine, Showa-Ku, Nagoya 466-8560, Japan. Tel.: 81-52-744-2674; fax: 81-52-744-2682; E-mail: thabeshi@med.nagoya-u.ac.jp.

Received February 22, 2001; revised June 29, 2001; accepted July $1,2001$.

Online publication: 7/19/01 at www.acnp.org/citations/ Npp071901151. which require the activation of the transcriptional and translational machinery (Goelet et al. 1986; Morgan and Curran 1991). Identification of the brain regions associated with both short-term and long-term memory is particularly important for a better understanding of the neural mechanism of learning and memory.

The $c$-fos oncogene is one of the immediate-early genes, and the gene product Fos is thought to activate late-effector genes by forming the heterodimeric AP-1 transcription factor (Morgan and Curran 1991). The expression of $c-f o s$ is induced by a variety of stimuli (Smith et al. 1992; Melia et al. 1994; Duncan et al. 1996) and after some forms of learning (McCabe and Horn 1994; Radulovic et al. 1998; Wan et al. 1999; Vann et al. 2000). Accordingly, Fos expression is considered a mechanism by which brief stimuli can trigger long-term changes in gene expression and alter the structural and functional properties of nerve cells (Goelet et al. 1986; Morgan and Curran 1991).

To identify potential anatomical substrates of spatial learning and memory in rats, we investigated the learn- 
ing-associated changes in Fos expression in rats that were subjected to the reference/working memory task with an eight-arm radial maze (Zou et al. 1998, 1999; Mizuno et al. 2000). Furthermore, to examine the possible causal relation between Fos expression and memory formation, we investigated the effects of an antisense oligonucleotide treatment directed against $c$-fos mRNA on spatial memory formation. Here, we show that Fos expression in the CA3 region of the dorsal hippocampus may be obligatory for spatial memory formation in the radial arm task.

\section{MATERIALS AND METHODS}

All experiments were performed in accordance with the Guidelines for Animal Experiments of the Nagoya University School of Medicine and the U.S. National Institutes of Health Guide for the Care and Use of Laboratory Animals. Male Wistar rats (7 weeks old; Charles River Japan, Yokohama, Japan) weighing $230 \pm 10 \mathrm{~g}$ at the beginning of experiments were used in the study. They were housed three per cage with free access to food and water under controlled laboratory conditions (a 12-h light/dark cycle with lights on at 9:00 a.m., $23 \pm$ $0.5^{\circ} \mathrm{C}, 50 \pm 0.5 \%$ humidity).

\section{Radial Arm Maze Task}

The radial arm maze used in this study consisted of eight arms, numbered from 1 to $8(48 \times 12 \mathrm{~cm})$, extending radially from a central area (32 $\mathrm{cm}$ in diameter), with a $5-\mathrm{cm}$ edge around the apparatus. Rats $(n=9)$ were trained for the reference/working memory task, five trials per day for 5 days, as described previously (Zou et al. 1998, 1999; Mizuno et al. 2000). Briefly, rats were well handled for 3 days in their home cages and then shaped for 4 days in the maze to run to the end of the arms and consume the bait, in groups of four rats. The bait was initially available throughout the maze but gradually was restricted to the food cup. After this shaping period, each animal was placed individually in the center of the maze and subjected to a reference and working memory task, where the same four arms (nos. 1, 2, 4, and 7) were baited for each training trial. The other four arms (nos. 3, 5, 6, and 8) were never baited. The training trial continued until all four baits in the food cups had been consumed or until $5 \mathrm{~min}$ had elapsed. Reference memory is regarded as a long-term memory for information that remains constant over repeated trials (memory for the positions of baited arms), whereas working memory is considered a short-term memory in which the information to be remembered changes in every trial (memory for the positions of arms that had already been visited in each trial; Olton et al. 1979). Thus, measures were made of the number of reference memory errors (entering an arm that was not baited) and working memory errors (entering an arm containing food but previously entered). A control group of animals $(n=3)$ was prepared as sham-trained rats; these were kept on a restricted diet as were the trained animals and put in the radial arm maze every day without maze training. Four food pellets were given for the sham-trained control group in the central area of the maze. Naive rats $(n=3)$ were kept with free access to food and water and were never placed in the radial arm maze. To determine learning-associated Fos expression, rats were trained for five trials per day with a 5-min intertrial interval for 5 days. After each training trial, rats were returned to their home cages. They were killed $2 \mathrm{~h}$ after the last training trials of days 1,3 , and 5 ( $n=3$ at each time point) for Fos immunohistochemistry. The sham-trained control rats $(n=3)$ were killed on day 3.

\section{Antisense Oligonucleotide Treatment}

Phosphorothiolated 15-mer sense and antisense oligonucleotides (antisense; 5'-GAA CAT CAT GGT CGT-3') were custom synthesized at SAWADY Technology (Tokyo, Japan) and dissolved in sterile pyrogen-free $0.9 \%$ saline. Rats were anesthetized with pentobarbital (50 $\mathrm{mg} / \mathrm{kg}$, i.p.) and placed in a stereotaxic apparatus. Sense $(n=11)$ or antisense c-fos oligonucleotide $(n=10)$ or saline $(n=9)$ was injected into the dorsal hippocampus bilaterally (A: $-3.8, \mathrm{~L}: \pm 2.5, \mathrm{~V}: 3.5$ ) according to the atlas of Paxinos and Watson (1982). Alternatively, rats received four injections of the sense $(n=6)$, antisense oligonucleotide $(n=8)$, or saline $(n=7)$ into the cingulate and motor cortex, two injections into the cingulate cortex (A: 2.7, L: $\pm 0.5, \mathrm{~V}: 2.5$ ), and two injections into the motor cortex (A: 0.7, L: $\pm 2.0, \mathrm{~V}: 2.0$ ). The oligonucleotide (5 nmol) or saline was injected in a volume of $1 \mu \mathrm{l}$ in each side over a 2-min period, and the injection cannula was left in place for an additional $5 \mathrm{~min}$ to allow for diffusion of oligonucleotide away from the injection site. The sequence of antisense oligonucleotide, dosage and duration of the action were based on published reports (Hooper et al. 1994; Morrow et al. 1999).

Twelve hours after the oligonucleotide treatment, the animals received 20 successive trials of the reference/ working memory task with a 5-min intertrial interval. They were killed $90 \mathrm{~min}$ after the last trial for Fos immunohistochemistry. In the second experiment, the maze training (20 successive trials) was started $10 \mathrm{~h}$ after the oligonucleotide treatment, and memory retention (five trials) was tested $24 \mathrm{~h}$ after the last training trial.

\section{Fos Immunohistochemistry}

Rats were deeply anesthetized with pentobarbital (50 $\mathrm{mg} / \mathrm{kg}$ ) and transcardially perfused with ice-cold phos- 
phate buffer $(0.1 \mathrm{M}, \mathrm{pH} 7.4)$ followed by $4 \%$ paraformaldehyde in phosphate buffer. The brains were removed, post-fixed in the same fixative for $2 \mathrm{~h}$, and then cryoprotected in $30 \%$ sucrose in phosphate buffer. The brains were cut into $50-\mu \mathrm{m}$ coronal sections on a cryostat, and free-floating sections were used for Fos immunohistochemistry. The sections were incubated with $5 \%$ goat serum and $0.3 \%$ Triton X-100 in $0.1 \mathrm{M}$ phosphate buffer and then incubated with rabbit anti-Fos antibody (1:2000; sc-52; Santa Cruz Biotechnology) for $48 \mathrm{~h}$ at $4^{\circ} \mathrm{C}$ with constant rotation. Sections were then washed with phosphate buffer containing $0.3 \%$ Triton X-100 and incubated with biotinylated goat anti-rabbit antibody at room temperature for $2 \mathrm{~h}$. Sections were washed and processed with avidin-biotinylated horseradish peroxidase complex (Vector ABC kit; Vector Laboratories), and the reaction was visualized using diaminobenzidine.

\section{Quantitative Analysis of c-Fos Immunohistochemistry}

To quantify the number of Fos-stained cells in the brain, we examined the sections with a computer-assisted image analysis system (C. Imaging Systems; Compix Inc., Mars, PA) attached to a light microscope (Olympus BX60-FLB-3; Olympus, Tokyo, Japan), as described previously (Yamada et al. 1996). Each group consisted of three rats at each time point. Both right and left hemispheres of 3-4 stions at each level of $+2.70,+0.70$, and $-3.80 \mathrm{~mm}$ from the bregma in each animal were examined for the counting of Fos-positive cells. Selected brain areas were as follows: prelimbic, cingulate, and motor cortex in sections at a level of +2.70 from the bregma; and cingulate, motor, and somatosensory cortex, dorsal and ventral caudate putamen, and the medial septal nucleus/nucleus of the diagonal band in sections at a level of +0.70 from the bregma. Additionally, regions $\mathrm{CA} 1, \mathrm{CA} 3$, and the dentate gyrus of the dorsal hippocampus, thalamic areas including the posterior thalamic nuclear group, ventroposterior thalamic nucleus, and paraventricular thalamic nucleus, as well as the posterior hypothalamic nucleus in sections at a level of -3.80 from the bregma were examined. The examined areas were from 0.5 to $1.3 \mathrm{~mm}^{2}$, depending on the brain regions.

\section{Statistical Analysis}

Results were expressed as the mean \pm SE. The significance of differences was determined by a one-way analysis of variance (ANOVA) followed by Bonferroni's test. Student's $t$-test was used for two-group comparisons. An ANOVA with repeated measures was conducted for analyzing data of the radial arm maze, and $p$ values of less than .05 were regarded as statistically significant.

\section{RESULTS}

\section{Fos Expression Induced by Spatial Learning in the Radial Maze}

To determine the relationship between memory formation and Fos expression, we trained rats for the reference and working memory task in the eight-arm radial maze, where the same four arms were baited for each training trial. Figure 1 shows the alteration of maze performance produced by repeated daily training (five trials per day). One-way ANOVA revealed a significant effect of training on reference $[\mathrm{F}(4,38)=52.479, p<$ $.0001]$ and working memory $[\mathrm{F}(4,38)=6.6191, p=$ .0004]. Both spatial reference and working memory were firmly formed by day 5 of training. Rats were killed for Fos immunohistochemistry $2 \mathrm{~h}$ after the last training trial on day 1,3 , or 5 . For comparison, two additional groups, the naive and sham-trained control groups, were prepared. The sham-trained control group was kept on a restricted diet, as were the trained animals, put on the radial arm maze everyday without actual maze training, and given four food pellets in every sham-training trial (Mizuno et al. 2000).

Representative photomicrographs of Fos staining in the cingulate and motor cortex and the dorsal hippocampus are shown in Figure 2, and summaries of fos

\section{a}

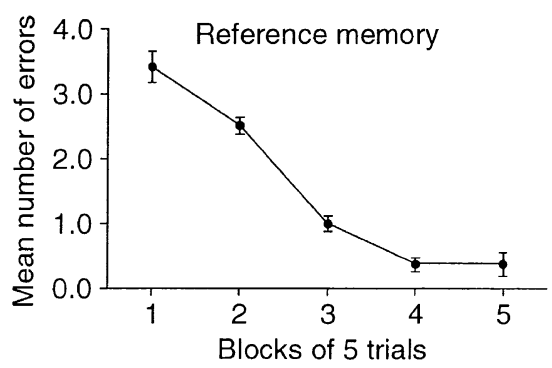

\section{b}

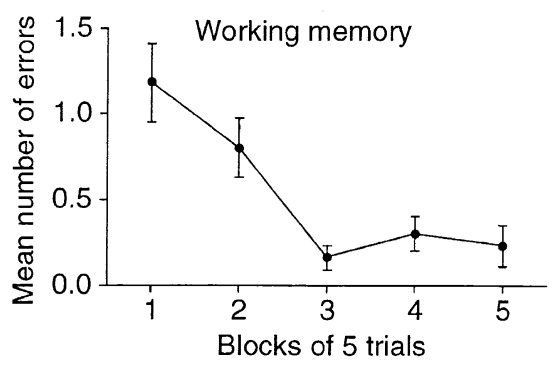

Figure 1. Spatial reference and working memory formation in rats. Rats were trained for the reference/working memory task, five trials per day for 5 days. Rats were killed $2 \mathrm{~h}$ after the last training trials on days 1,3 , and 5 for Fos immunohistochemistry. There were 12 animals on day 1, 9 on days 2 and 3 , and 6 on days 4 and 5 . Repeated training significantly reduced the number of reference memory $[\mathrm{F}(4,42)=52.479, p<.0001]$ (a) and working memory errors $[\mathrm{F}(4,42)=6.6191, p=.0004](\mathrm{b})$. 


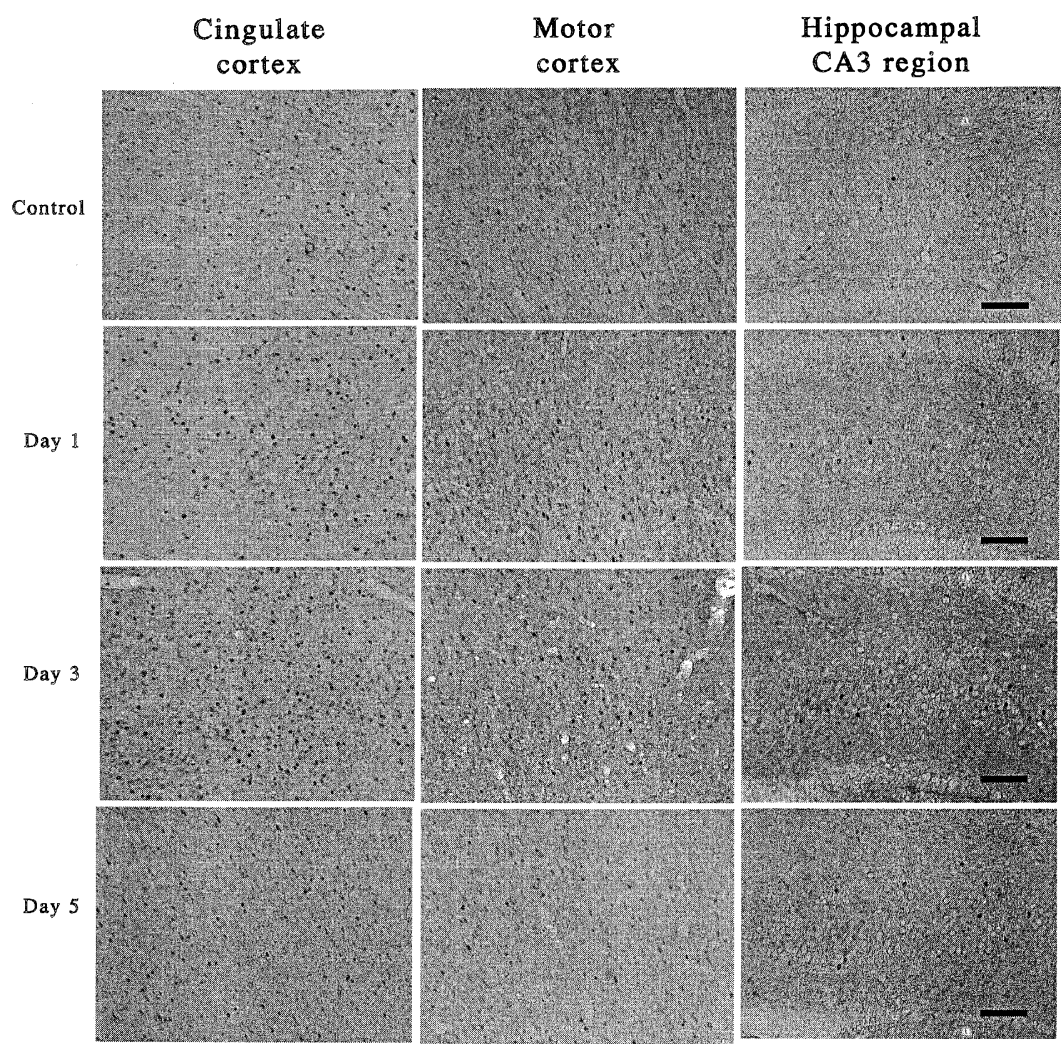

Figure 2. Representative photomicrographs of Fos immunostaining of the cingulate and motor cortex, CA1 and CA3 region, and the dentate gyrus of the hippocampus in rats subjected to the radial arm maze task. Scale bar, $100 \mu \mathrm{m}$.

expression observed in all brain areas are shown in Figure 3. Analysis of the density of Fos-positive cells revealed a significant difference between naive and control groups in the prelimbic cortex $(p<.01)$, cingulate cortex $(p<.05)$, motor cortex $(p<.05)$, somatosensory cortex $(p<.05)$, medial septal nucleus/nucleus of the diagonal band $(p<.001)$, and the thalamic area (including the posterior thalamic nuclear group, ventroposterior thalamic nucleus, and paraventricular thalamic nucleus) $(p<.05)$. There was no difference between naive and control groups in other brain regions measured, such as caudate putamen, posterior hypothalamic nucleus, and the hippocampus (Figure 3). The radial arm maze training caused a significant alteration of the density of Fos-positive cells in the prelimbic cortex $[\mathrm{F}(3,8)=$ $12.786, p=.0020]$, cingulate cortex $[\mathrm{F}(3,8)=14.499, p=$ $.0013]$, motor cortex $[\mathrm{F}(3,8)=33.613, p<.0001]$, somatosensory cortex $[\mathrm{F}(3,8)=7.1393, p=.0119]$, medial septal nucleus/nucleus of the diagonal band $[\mathrm{F}(3,8)=$ $10.200, p=.0041]$, and the CA3 region of the dorsal hippocampus $[\mathrm{F}(3,8)=15.242, p<.0011]$. No changes were found in the caudate putamen, thalamus, posterior hypothalamic nucleus, and the CA1 region and dentate gyrus of the hippocampus. Post-hoc analyses revealed that in the prelimbic, cingulate, and somatosensory cortex as well as in the CA3 region of the hippocampus, the maze training did not affect the numbers of Fospositive cells on day 1 , but they were significantly increased on day 3 and then returned to control level on day 5 . In the motor cortex, a significant increase in the number of Fos-positive cells was observed on day 1 , which was further increased on day 3 and then decreased to the control level on day 5. Fos expression in the medial septal nucleus/nucleus of the diagonal band was also increased on day 1 but then returned to the control level on days 3 and 5 (Figure 3).

\section{Effect of Intrahippocampal Microinjections of Antisense c-fos Oligonucleotide on Spatial Memory Formation}

To assess the possible causal relationship between Fos expression and spatial memory formation, we trained rats that received microinjections of the antisense oligonucleotide into either the dorsal hippocampus or the cingulate and motor cortex for the reference and working memory task. Figures $4 \mathrm{a}-\mathrm{c}$ illustrate the maze performance in rats that received microinjections of the oligonucleotide into the hippocampus.

Significant effects of group $[\mathrm{F}(2,27)=31.552, p<$ $.0001]$ and trial $[\mathrm{F}(3,81)=53.457, p<.0001]$ but not group by trial interaction $[\mathrm{F}(6,81)=0.867, p=.5232]$ were observed in reference memory formation in rats that had previously received intrahippocampal injections of the oligonucleotide. The same effects were also observed in working memory formation [group effect, $\mathrm{F}(2,27)=28.136, p<.0001$; trial effect, $\mathrm{F}(3,81)=12.319$, $p<.0001$; group by trial effect, $\mathrm{F}(6,81)=1.389, p=$ 

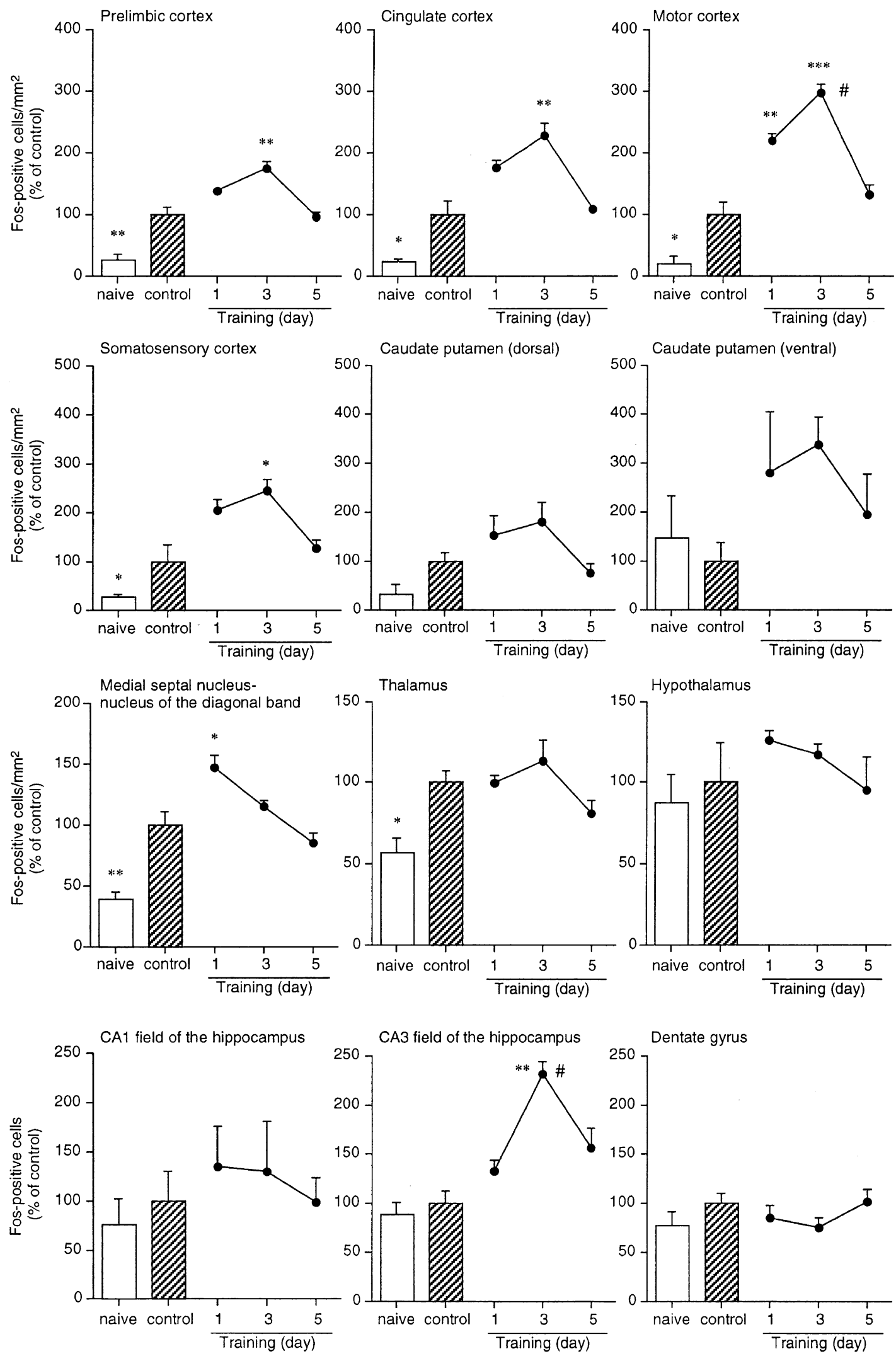

Figure 3. Changes in the density of Fos-positive cells in various brain areas during the spatial reference/working memory training. Rats were trained for the reference/working memory task, five trials per day for 5 days. They were killed $2 \mathrm{~h}$ after the last training trial on days 1,3, and 5. Data are expressed as a percentage of the sham-training control group. Each value represents the mean $\pm \mathrm{SE}$ of three rats. ${ }^{*} p<.05,{ }^{* *} p<.01,{ }^{* * *} p<.001$ versus control. $\# p<.05$ versus training group on day 1. 

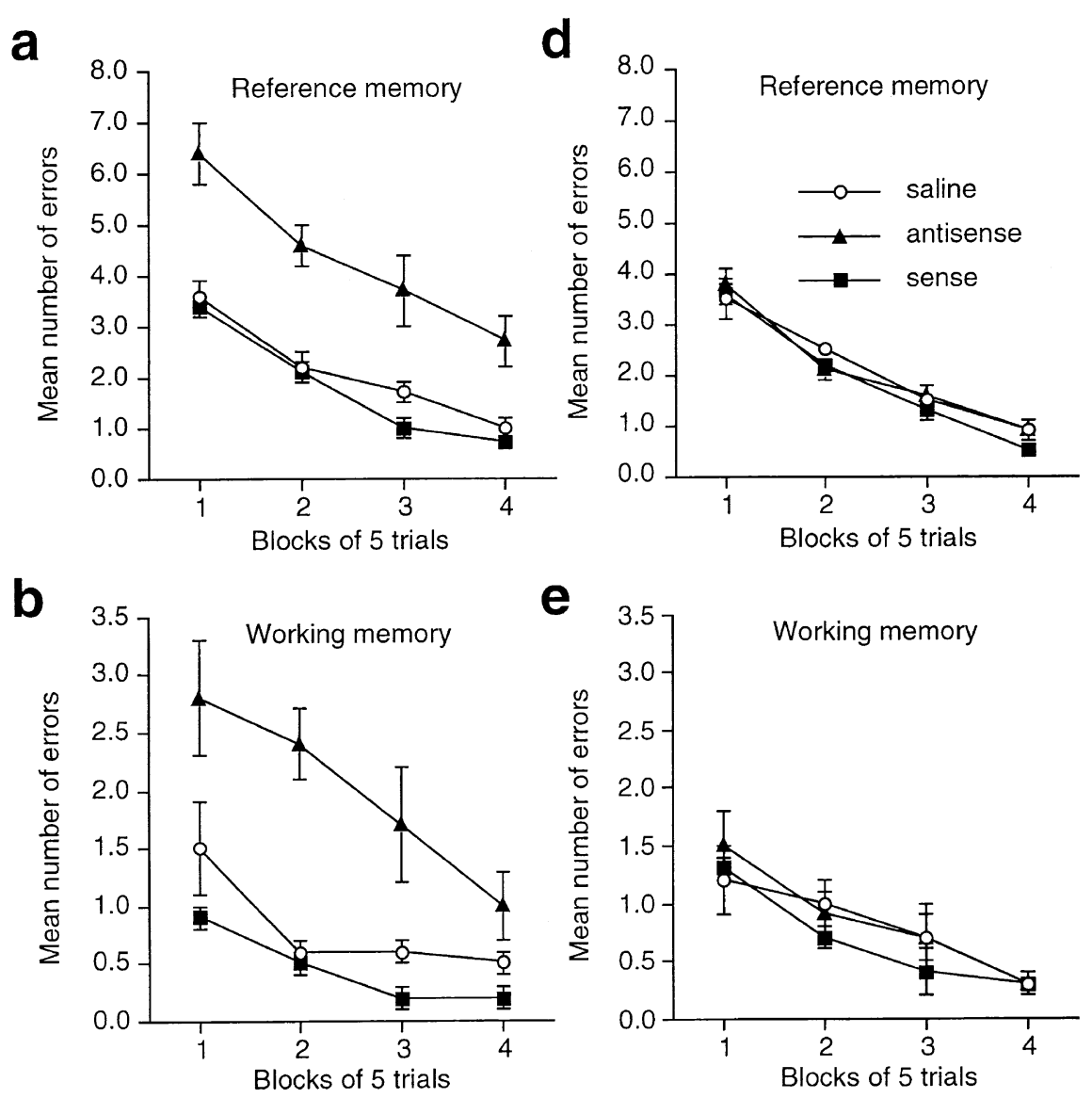

Figure 4. The effects of microinjections of antisense c-fos oligonucleotide into the dorsal hippocampus $(\mathbf{a}-\mathbf{c})$ or the cingulate and motor cortex (d-f) on spatial memory formation. a and $\mathbf{d}$ ) reference memory; $\mathbf{b}$ and $\mathbf{e}$ ) working memory; $\mathbf{c}$ and $\mathbf{f}$ ) locomotor function. Rats were injected with either saline or antisense or sense oligonucleotide and then received 20 successive training trials with a 5-min intertrial interval $12 \mathrm{~h}$ after the treatment. Each value represents the mean \pm SE of $8-11$ rats.
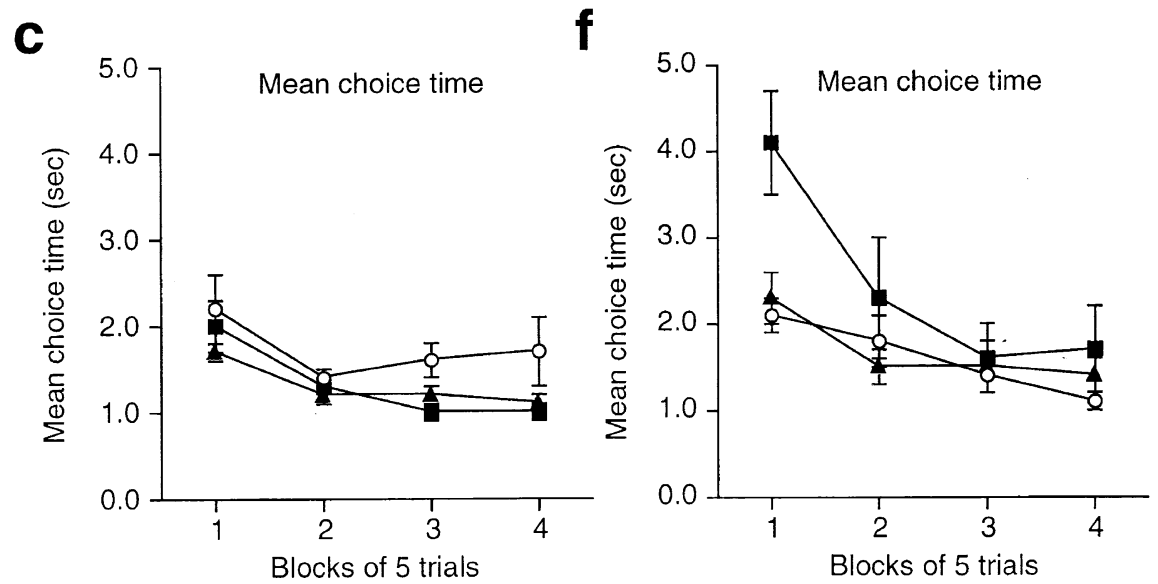

.2290]. Post-hoc analysis indicated that the antisense oligonucleotide injections into the hippocampus significantly impaired both spatial reference $(p<.001)$ and working memory formation $(p<.001)$, although the sense oligonucleotide treatment had no effect (Figures $4 a$ and $b$ ). We also analyzed the effect of the antisense oligonucleotide on locomotor function and appetite, which potentially affect the maze performance. As shown in Figure 4c, the mean time spent for each choice did not differ among the three groups of rats [group effect,: $\mathrm{F}(2,27)=2.767, p=.0807$; trial effect, $\mathrm{F}(3,81)=$ $11.903, p<.0001$; group by trial effect, $\mathrm{F}(6,81)=0.951$, $p=.4637]$. There was also no apparent difference among the groups in food consumption during the trials. It is unlikely, therefore, that the impairment of maze performance in rats that received the antisense c-fos oligonucleotide into the hippocampus results from motor dysfunction or an altered motivational state.

In the second experiment, the maze training was started $10 \mathrm{~h}$ after microinjections of the antisense c-fos oligonucleotide into the hippocampus. As in the first experiment, there were significant effects in reference memory [group effect, $\mathrm{F}(2,19)=18.129, p<.0001$; trial effect, $\mathrm{F}(3,57)=48.541, p<.0001$, group by trial interac- 
tion, $\mathrm{F}(6,57)=1.690, p=.1401]$ and working memory formation [group effect, $\mathrm{F}(2,27)=28.136, p<.0001$; trial effect, $\mathrm{F}(3,81)=12.319, p<.0001$; group by trial interaction, $\mathrm{F}(6,81)=1.389, p=.2290]$. Post-hoc analysis indicated that the antisense oligonucleotide injections into the hippocampus significantly impaired both spatial reference $(p<.001)$ and working memory formation $(p<$ .001 ), although the sense oligonucleotide treatment had no effect (data not shown). To examine the effect of microinjections of the antisense c-fos oligonucleotide into the hippocampus on memory retention and consolidation, we retested performance in the radial arm maze 24 $\mathrm{h}$ after the last training trial. The mean numbers of reference and working memory errors in the retention test did not differ from those in the last five trials of 20 successive training trials in saline, antisense, and sense oligonucleotide-treated groups (data not shown).

\section{Effect of Intracortical Microinjections of Fos Antisense Oligonucleotide on Spatial Memory Formation}

We also examined the effect of inhibition of Fos expression in the cingulate and motor cortex on spatial memory formation. In contrast to the marked effects in the hippocampus, neither spatial reference nor working memory formation was affected by microinjections of the antisense oligonucleotide into the cingulate and motor cortex (Figures $4 \mathrm{~d}-\mathrm{f}$ ). There were also no apparent differences in locomotor activity and food consumption during the training trials among three groups of rats that received microinjections of saline, antisense, or sense oligonucleotide.

\section{Effect of the Antisense Oligonucleotide Treatment on Learning-Associated Fos Expression}

Finally, the effect of the antisense oligonucleotide treatment on Fos expression was confirmed by Fos immunohistochemistry (Figure 5). Fos expression in the hippocampus and cingulate and motor cortex was markedly diminished by microinjections of the antisense oligonucleotide into these brain areas as compared with those in saline-injected rats. Quantitative analysis of Fos immunohistochemistry revealed that the antisense treatment significantly reduced the density of Fos-positive cells in all brain areas examined to $<50 \%$ of salineinjected control groups. The expression was not affected by the sense oligonucleotide treatment (Figure 6).

\section{DISCUSSION}

The present findings with Fos immunohistochemistry demonstrate that the cerebral cortices and the CA3 region of the dorsal hippocampus are activated during the course of the spatial reference/working memory task in the radial maze. These results are consistent with the well-established role of these brain areas in learning and memory (Olton et al. 1979; Morris et al. 1982; Kesner et al. 1987; Jarrard 1993; Rudy and Sutherland 1995). Some studies have shown that the dorsal hippocampal formation is more important than the ventral formation for spatial learning (Jung et al. 1994; Moser et al. 1995). Although we did not examine the Fos expression in the ventral hippocampus in the present study, our findings suggest that neurons in the dorsal hippocampus are activated, not uniformly, but in a brain region-specific manner.

It should be determined whether the observed changes in Fos expression are directly related to learning and memory per se. Some concerns have been raised regarding the contribution of novelty and stress to Fos expression during the learning (Asanuma and Ogawa 1994; Dragunow 1996). To reduce the effect of these factors, animals were extensively habituated to the test environment and apparatus before starting the actual learning trials, and sham-trained control rats were prepared for the comparison. It is important to note that Fos expression in the cortical areas such as prelimbic, cingulate, and the somatosensory cortex as well as in the CA3 region of the hippocampus increased on day 3 but not on day 1, although stress levels are considered to decrease on day 3 compared with day 1 . Therefore, it is unlikely that Fos expression in the cerebral cortices and hippocampus during the maze training is due to stress and other aspects of the training situation. A stress effect may be involved in the medial septal nucleus/nucleus of the diagonal band because increased Fos expression was observed on day 1 but not other days. Locomotor activity in the maze may also contribute to the Fos expression in the motor cortex.

Some studies have suggested the importance of the CA3 region of the hippocampus in memory formation (Hess et al. 1995; Gall et al. 1998; Zhao et al. 2000). For instance, Gall et al. (1998) have demonstrated in twoodor discrimination learning that preferential activation of the CA3 region is uniquely associated with initial learning of an odor pair, whereas predominant activation of the CA1 occurred on exploration of a novel field and with overtrained animals' responding to odors. Also, upregulation of $c$-src (a nonreceptor tyrosine protein kinase) mRNA was observed in the CA3 region of the hippocampus after spatial learning in a water maze, whereas learning-associated changes were not observed in the CA1 region or dentate gyrus (Zhao et al. 2000).

To investigate the role of Fos expression in spatial memory formation in the cerebral cortices and the hippocampal CA3 region, the antisense oligonucleotide was directly injected into these brain regions. For these experiments, we used a single-session protocol of 20 tri- 


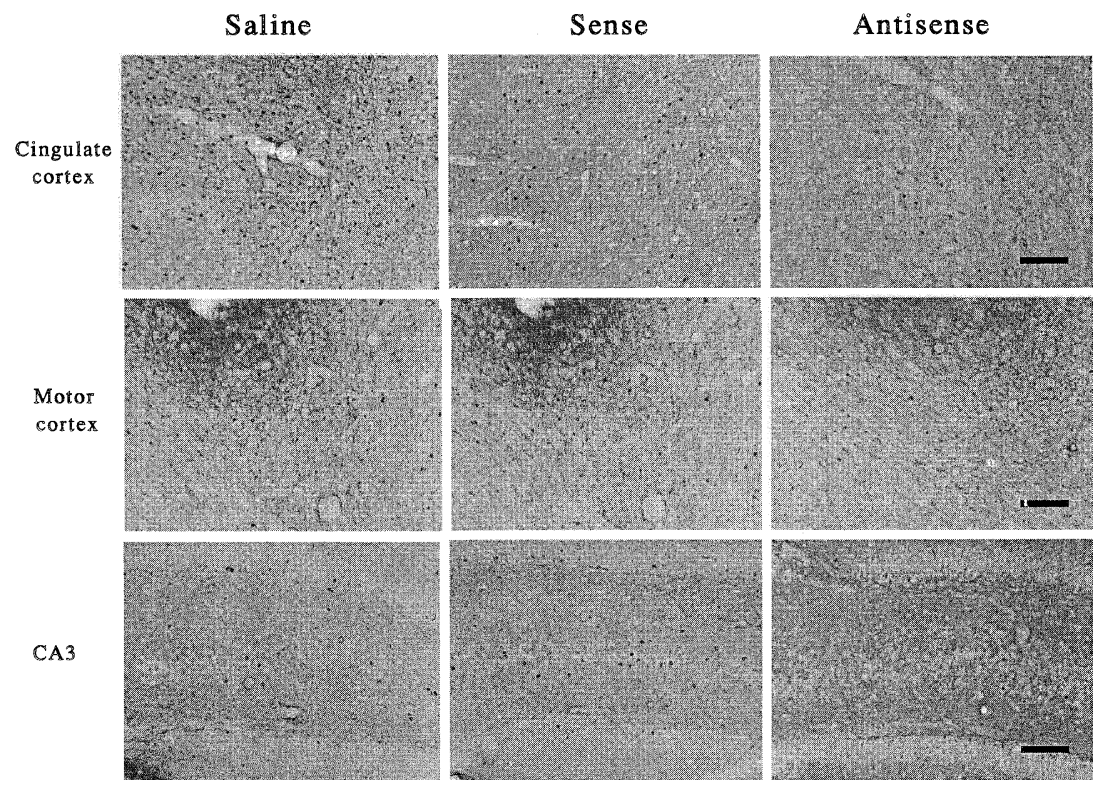

Figure 5. Representative photomicrographs of Fos immunostaining of the cingulate and motor cortex and the CA3 region of the hippocampus in proximate sites of injection of either saline or sense or antisense c-fos oligonucleotide. Scale bar, $100 \mu \mathrm{m}$.

als / day as opposed to the daily training trials (5 trials / day for 5 days) used in the first experiment to avoid brain damage due to repeated insertion of the microinjection needle, which is necessary for the daily training protocol. Thus, there is a possibility that an alteration of training protocol may affect the kinetics of memory process and of the learning-associated Fos expression.

Consistent with previous studies (Hooper et al. 1994; Morrow et al. 1999), the injection of the antisense, but not sense, oligonucleotide effectively inhibited the local Fos expression near the injection sites (Figures 5 and 6). Inhibition of Fos expression in the hippocampus resulted in a significant increase in the number of reference and working memory errors, although injections of the antisense oligonucleotide into the cingulate and motor cortex had no effect. It is unlikely that the phosphorothiolated oligonucleotide caused damage or toxicity to neurons in the CA3 regions of the hippocampus and therefore resulted in the disruption of the maze performance because the phosphorothiolated sense oligonucleotide did not have the same effect. Accordingly, a possible interpretation of the data is that Fos expression in the CA3 region of the dorsal hippocampus is necessary for spatial memory formation.

A close examination of the data presented in Figure 4 indicates that rats treated with the antisense oligonucleotide into the hippocampus showed a significant reduction in the number of both reference and working memory errors over blocks of trials. The parallel decline of errors as compared with the sense- and saline-treated animals revealed a performance, not memory, impairment, suggesting that the antisense treatment may affect non-mnemonic components. In fact, a previous study reported global disruptions in behavior with the use of antisense oligonucleotide directed against c-fos
(Hooper et al. 1994). However, no alteration in time spent for each choice, locomotor activity, or food intake during the maze test was observed under our experimental conditions in rats treated with the antisense c-fos oligonucleotide. Therefore, it is unlikely that impairment of maze performance in the antisense-treated rats is due to disruption of non-mnemonic components. It remains possible, however, that other relevant aspects of behaviors that were not tested may be affected by the antisense oligonucleotide treatment.

Previous studies have demonstrated that the antisense c-fos oligonucleotide inhibits Fos production in the brain induced by various stimuli when it was injected 4 to $12 \mathrm{~h}$ before the test stimulus (Chiasson et al., 1992; Heilig et al., 1993; Hooper et al., 1994; Hunter et al., 1995; Morrow et al., 1999), whereas the same antisense c-fos oligonucleotide failed to suppress amphetamine-induced Fos expression in the striatum when it was injected $22 \mathrm{~h}$ before amphetamine injection (Chiasson et al., 1992). In the present study, the maze training was started $12 \mathrm{~h}$ after the antisense treatment, and it took $\sim 2 \mathrm{~h}$. Therefore, it is unclear whether Fos production was inhibited by the antisense treatment during the entire time of training. One might consider that performance in the antisense-treated rats could be almost the same as in the saline-treated control animals at the end of maze training if activity of the antisense oligonucleotide was diminished during the training. To address this issue, we set up the second experiment, in which the maze training was started $10 \mathrm{~h}$ after microinjections of the antisense c-fos oligonucleotide into the hippocampus. As in the first experiment, it was found that the antisense treatment slowed the learning of the spatial task performance but did not prevent learning. The antisense-treated animals improved in perfor- 


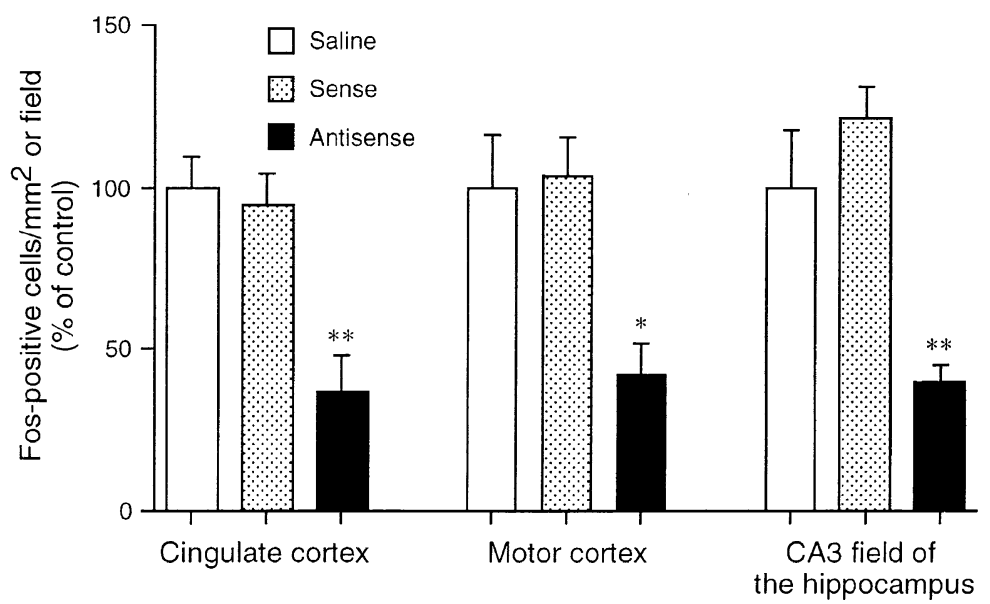

Figure 6. The effects of microinjections of antisense c-fos oligonucleotide into the dorsal hippocampus, cingulated cortex, or motor cortex on the density of Fos-positive cells in rats that received spatial reference/working memory training. Rats were injected with either saline or antisense or sense oligonucleotide and then received 20 successive training trials with a 5-min intertrial interval $12 \mathrm{~h}$ after the treatment. Each value represents the mean \pm SE of five to six rats. ${ }^{*} p<.05,{ }^{* *} p<$ .01 versus saline-treated control.

mance so much that by the end of the 20 trials they were performing at almost the same levels as the control group. Moreover, memory retention and consolidation tested $24 \mathrm{~h}$ after the last training were not affected by the antisense treatment into the hippocampus. Collectively, it is likely that inhibition of Fos production with antisense oligonucleotide in the CA3 region of the hippocampus inhibits but not prevents spatial learning in the radial arm maze task.

There may be some compensatory mechanisms secondary to the inhibition of hippocampal Fos expression that allow learning to take place, albeit at a slightly lower rate in the antisense-treated rats. For instance, we have previously demonstrated that memory impairment in rats with an excitotoxic lesion of the hippocampus can be recovered by repeated training (Zou et al., 1999). Thus, although further experiments are required to elucidate a causal relationship, our present findings suggest an important role of Fos expression in the CA3 region of the hippocampus in spatial memory formation. We do not exclude the possibility that cerebral cortex is also involved in spatial memory formation, because we injected the oligonucleotide into only four sites of the cingulate and motor cortex, by which the extent of suppression of Fos expression may have been insufficient to impair spatial memory formation.

We have been using the reference/working memory task in the radial arm maze to clarify the neuronal mechanisms of each category of memory (Zou et al. 1998, 1999; Mizuno et al. 2000). The antisense $c$-fos oligonucleotide treatment into the dorsal hippocampus impaired not only long-term memory (reference memory) but also short-term working memory (Figure 4). It is tempting to speculate that inhibition of Fos expression in the hippocampus impairs reference memory formation by inhibiting the expression of a number of target genes that play important roles in long-term changes in synaptic connections (Goelet et al. 1986; Morgan and Curran 1991; Dragunow 1996). On the other hand, it is less likely that Fos expression has a direct effect on spatial working memory when considering the given role of Fos protein, because the information on working memory changes for every choice of arm in each training trial. Rather, it is considered that inhibition of working memory formation induced by the antisense treatment is due to a secondary effect of the impairment of reference memory formation.

In conclusion, Fos expression in the cerebral cortex and CA3 region of the dorsal hippocampus is increased during the spatial learning in the radial arm maze, and this region-specific increase in the hippocampus may be obligatory for spatial memory formation. Our results support the hypothesis that the inducible transcription factor $c-f o s$ is essential for encoding spatial memory.

\section{ACKNOWLEDGMENTS}

This study was supported in part by a Grant-in-Aid for Scientific Research (No.12670085), a COE Grant, and Special Coordination Funds for Promoting Science and Technology, Target-Oriented Brain Science Research Program, from the Ministry of Education, Culture, Sports, Science and Technology of Japan.

\section{REFERENCES}

Asanuma M, Ogawa N (1994): Pitfalls in assessment of c-fos mRNA expression in the brain: Effects of animal handling. Rev Neurosci 5:171-178

Burns ME, Augustine GJ (1995): Synaptic structure and function: Dynamic organization yields architectural precision. Cell 83:187-194

Chiasson BJ, Hooper ML, Murphy PR, Robertson HA (1992): Antisense oligonucleotide eliminates in vivo expression of c-fos in mammalian brain. Eur J Pharmacol 227:451-453

Dragunow M (1996): A role for immediate-early transcription factors in learning and memory. Behav Genet 26:293-299 
Duncan GE, Knapp DJ, Breese GR (1996): Neuroanatomical characterization of Fos induction in rat behavioral models of anxiety. Brain Res 713:79-91

Edwards FA (1995): Anatomy and electrophysiology of fast central synapses lead to a structural model for longterm potentiation. Physiol Rev 75:759-787

Gall CM, Hess US, Lynch G (1998): Mapping brain networks engaged by, and changed by, learning. Neurobiol Learn Memo 70:14-36

Goelet P, Castellucci VF, Schacher S, Kandel ER (1986): The long and the short of long-term memory-A molecular framework. Nature 322:419-423

Heilig M, Engel JA, Söderpalm B (1993): C-fos antisense in the nucleus accumbens blocks the locomotor stimulant action of cocaine. Eur J Pharmacol 236:339-340

Hess US, Lynch G, Gall CM (1995): Changes in c-fos expression in rat brain during odor discrimination learning: Differential involvement of hippocampal regions CA1 and CA3. J Neurosci 15:4786-4795

Hooper ML, Chiasson BJ, Robertson HA (1994): Infusion into the brain of an antisense oligonucleotide to the immediate-early gene c-fos suppresses production of fos and produces a behavioral effect. Neuroscience 63:917-924

Hunter JC, Woodburn VL, Durieux C, Pettersson EKE, Poat JA, Hughes J (1995): C-fos antisense oligodeoxynucleotide increases formalin-induced nociception and regulates preprodynorphin expression. Neuroscience 65:485-492

Jarrard LE (1993): On the role of the hippocampus in learning and memory in the rat. Behav Neural Biol 60:9-26

Jung MW, Wiener SI, McNaughton BL (1994): Comparison of spatial firing characteristics of units in dorsal and ventral hippocampus of the rat. J Neurosci 14:7347-7356

Kesner RP, Dimattia BV, Crutcher KA (1987): Evidence for neocortical involvement in reference memory. Behav Neural Biol 47:40-53

Kleim JA, Lussnig E, Schwarz ER, Comery TA, Greenough WT (1996): Synaptogenesis and FOS expression in the motor cortex of the adult rats after motor skill learning. J Neurosci 14:4529-4535

McCabe BJ, Horn G (1994): Learning-related changes in Foslike immunoreactivity in the chick forebrain after imprinting. Proc Natl Acad Sci USA 91:11417-11421

Melia KR, Ryabinin AE, Schroeder R, Bloom FE, Wilson MC (1994): Induction and habituation of immediate early gene expression in rat by acute and repeated restraint stress. J Neurosci 14:5929-5938

Mizuno M, Yamada K, Olariu A, Nawa H, Nabeshima T (2000): Involvement of BDNF in spatial memory formation and maintenance in a radial arm maze test in rats. $\mathrm{J}$ Neurosci 20:7116-7121
Morgan JI, Curran T (1991): Stimulus-transcription coupling in the nervous system: Involvement of the inducible protooncogenes fos and jun. Ann Rev Neurosci 14:421-451

Morris RGM, Garrud P, Rawlins JNP, O'Keefe J (1982): Place navigation in rats with hippocampal lesions. Nature 297:681-683

Morrow BA, Elsworth JD, Inglis FM, Roth RH (1999): An antisense oligonucleotide reverses the footshockinduced expression of Fos in the rat medial prefrontal cortex and the subsequent expression of conditioned fear-induced immobility. J Neurosci 19:5666-5673

Moser M-B, Moser EI, Forrest E, Andersen P, Morris RGM (1995): Spatial learning with minislab in the dorsal hippocampus. Proc Natl Acad Sci USA 92:9697-9701

Olton DS, Becker JT, Handelmann GE (1979): Hippocampus, space, and memory. Behav Brain Sci 2:313-365

Paxinos G, Watson C (1982): The Rat Brain in Stereotaxic Coordinates. New York, Academic Press

Radulovic J, Kammermeier J, Spiess J (1998): Relationship between fos production and classical fear conditioning: Effects of novelty, latent inhibition, and unconditioned stimulus preexposure. J Neurosci 18:7452-7461

Rudy JW, Sutherland RJ (1995): Configural association theory and the hippocampal formation: An appraisal and reconfiguration. Hippocampus 5:375-389

Smith MA, Banerjee S, Gold PW, Glowa J (1992): Induction of c-fos mRNA in rat brain by conditioned and unconditioned stressors. Brain Res 578:135-141

Vann SD, Brown MW, Erichsen JT, Aggleton JP (2000): Fos imaging reveals differential patterns of hippocampal and parahippocampal subfield activation in rats in response to different spatial memory tests. J Neurosci 20:2711-2718

Wan H, Aggleton JP, Brown MW (1999): Different contributions of the hippocampus and perirhinal cortex to recognition memory. J Neurosci 19:1142-1148

Yamada K, Noda Y, Komori Y, Sugihara H, Hasegawa T, Nabeshima $T$ (1996): Reduction in the number of NADPH-diaphorase-positive cells in the cerebral cortex and striatum in aged rats. Neurosci Res 24:393-402

Zhao W, Cavallaro S, Gusev P, Alkon DJ (2000): Nonreceptor tyrosine protein kinase pp60c-src in spatial learning: Synapse-specific changes in its gene expression, tyrosine phosphorylation, and protein-protein interactions. Proc Natl Acad Sci USA 97:8098-8103

Zou L-B, Yamada K, Tanaka K, Kameyama T, Nabeshima T (1998): Nitric oxide synthase inhibitors impair reference memory formation in a radial arm maze task in rats. Neuropharmacology 37:323-330

Zou L-B, Yamada K, Sasa M, Nabeshima T (1999): Two phases of behavioral plasticity in rats following unilateral excitotoxic lesion of the hippocampus. Neuroscience 92:819-826 